\title{
Time-dependent association of glucocorticoids with adverse outcome in community-acquired pneumonia: a 6-year prospective cohort study
}

Manuela Nickler ${ }^{1}$, Manuel Ottiger ${ }^{1}$, Christian Steuer ${ }^{2}$, Alexander Kutz ${ }^{1}$, Mirjam Christ-Crain ${ }^{3,4}$, Werner Zimmerli' Robert Thomann ${ }^{6}$, Claus Hoess ${ }^{7}$, Christoph Henzen ${ }^{8}$, Luca Bernasconi ${ }^{2}$, Andreas Huber ${ }^{2}$, Beat Mueller ${ }^{1,4}$, and Philipp Schuetz ${ }^{1,4^{*}}$ for the ProHOSP Study Group

\begin{abstract}
Background: The hypothalamic-pituitary-adrenal stress axis plays a crucial role in community-acquired pneumonia (CAP), with high cortisol being associated with disease severity and corticosteroid treatment resulting in earlier time to recovery. Our aim in the present study was to compare different glucocorticoid hormones, including cortisol, 11-deoxycortisol, cortisone, and corticosterone, regarding their association with short- and long-term adverse outcomes in a well-defined CAP cohort.

Methods: We prospectively followed 285 patients with CAP from a previous Swiss multicenter trial for a median of 6.1 years and measured different admission glucocorticoid serum levels by liquid chromatography coupled with tandem mass spectrometry. We used adjusted Cox regression models to investigate associations between admission hormone levels and all-cause mortality at different time points.

Results: Mortality was 5.3\% after 30 days and increased to $47.3 \%$ after 6 years. High admission cortisol was associated with adverse outcome after 30 days (adjusted OR 3.85, 95\% Cl 1.10-13.49, $p=0.035$ ). In the long term (i.e.), however, high admission cortisol was associated with better survival (adjusted HR after 3 years $0.53,95 \% \mathrm{Cl} 0$. 32-0.89, $p=0.017$; adjusted HR after 6 years $0.57,95 \% \mathrm{Cl} 0.36-0.90, p=0.015)$. Compared with 11-deoxycortisol, cortisone, and corticosterone, cortisol showed the highest association with mortality.

Conclusions: Among different glucocorticoid hormones, cortisol showed the highest association with mortality in CAP. Whereas a more pronounced glucocorticoid stress response on hospital admission was associated with higher short-term adverse outcome, long-term outcome was favorable in these patients. These data should support the correct interpretation of glucocorticoid blood data.
\end{abstract}

Keywords: Community-acquired pneumonia, Glucocorticoid hormones, Cortisol, 11-Deoxycortisol, Cortisone, Corticosterone, Mortality/outcome prediction, Disease severity, Pneumonia severity index

\footnotetext{
* Correspondence: schuetzph@gmail.com

${ }^{1}$ Medical University Department, Division of General Internal and Emergency

Medicine, Kantonsspital Aarau, Aarau, Switzerland

${ }^{4}$ Medical Faculty, University of Basel, Basel, Switzerland

Full list of author information is available at the end of the article
}

\section{Biomed Central}

(c) The Author(s). 2017 Open Access This article is distributed under the terms of the Creative Commons Attribution 4.0 International License (http://creativecommons.org/licenses/by/4.0/), which permits unrestricted use, distribution, and reproduction in any medium, provided you give appropriate credit to the original author(s) and the source, provide a link to the Creative Commons license, and indicate if changes were made. The Creative Commons Public Domain Dedication waiver (http://creativecommons.org/publicdomain/zero/1.0/) applies to the data made available in this article, unless otherwise stated. 


\section{Background}

Activation of the hypothalamic-pituitary-adrenal (HPA) axis and stimulation of the central noradrenergic stress system by cytokines and other mediators are major pathophysiological adaptations in response to infection and inflammation [1-4]. Appropriate activation of the HPA axis during illness is crucial for survival and mirrors the stress level. The effects of cortisol include protection against excessive inflammatory reaction, acute supply of energy, and improvement in hemodynamic status [5, 6].

Endogenous adrenocortical function is of prognostic value in critical illness and sepsis [7-14], of which community-acquired pneumonia (CAP) is a major source [15-17]. Morbidity in CAP is still high also, owing to higher risk for cardiovascular events [18, 19]. Researchers in multiple studies have investigated the adrenal steroid metabolism in relation to CAP-associated outcomes [14, 20-25]. In observational trials, researchers have reported increased admission cortisol levels to be independent predictors of disease severity and short-term mortality in patients across the spectrum of mild to severe CAP [20-24]. Interestingly, the predictive accuracy of free cortisol was not superior to that of total cortisol, independent of serum albumin levels [20]. The prognostic accuracy of cortisol levels in short-term mortality prediction was equal to that of the pneumonia severity index (PSI), but better than the CURB-65 score (confusion of new onset, blood urea nitrogen $>7 \mathrm{mmol} / \mathrm{L}$, respiratory rate $\geq 30$ breaths per minute, systolic blood pressure $<90 \mathrm{mmHg}$ or diastolic blood pressure $\leq 60$, and age $\geq 65$ years) and routinely measured laboratory parameters such as C-reactive protein (CRP), procalcitonin (PCT), or white blood cell count [20, 21, 23]. In addition, in severe CAP, baseline serum cortisol levels were shown to be independent predictors of mortality with superior predictive ability compared with stimulated cortisol with corticotropin or $\Delta$-cortisol [24]. Despite evidence regarding short-term outcomes, less is known about the predictive potential of glucocorticoid levels in regard to long-term mortality. Aside from that, most studies have been focused on cortisol only, and data regarding other human glucocorticoids, such as 11deoxycortisol, cortisone, and corticosterone, are largely lacking. Identification of patients at risk for adverse outcome after an index hospitalization for CAP may optimize in-hospital and post-acute care strategies to improve survival. A better description of metabolite signatures in CAP may improve pathophysiological understanding and provide new targets for a more personalized therapy. In the present study, our aim was to study different compounds of the glucocorticoid pathway, including cortisol, 11-deoxycortisol, cortisone, and corticosterone, regarding their association with short- and long-term adverse outcomes in a well-defined CAP cohort.

\section{Methods}

\section{Study design and setting}

This is a secondary analysis of data from a prospective, randomized, controlled multicenter trial performed at six Swiss secondary or tertiary care centers between October 2006 and March 2008 [26]. A detailed study protocol of the initial trial has been published elsewhere [27]. Briefly, from a total of 1825 potential patients, 925 subjects with CAP were included. The aim of the initial trial was to assess the efficacy and safety of a PCT-guided antibiotic therapy compared with standard guidelines without using PCT data [28-30] in patients with CAP and other lower respiratory tract infections [31]. The study protocol was approved by the ethics committee of the University of Basel as well as by all local ethics committees, and written informed consent was provided by all participants for the initial trial, including agreement to use their data anonymized in secondary analyses.

\section{Selection and assessment of participants}

Inclusion criteria were age $>18$ years and final diagnosis of CAP with an infiltrate on a chest $x$-ray [31]. Exclusion criteria were language restriction or dementia precluding patients from providing written informed consent, presence of a terminal condition, and intravenous drug abuse. For reasons of the initial antibiotic stewardship trial, patients were excluded for severe immunosuppression or for long-term antibiotic therapy on admission independent of the current infection (e.g., in case of endocarditis or chronic infections), whereas a requirement of corticosteroids or short-term antibiotic pretreatment was allowed. All patients included in the analysis were inpatients.

Patient assessment included clinical and biochemical evaluation upon admission to the emergency department (ED) and throughout the period of hospitalization. The standardized baseline characteristics comprised medical history, comorbidities (identified either through patient self-report or medical chart review), vital signs including fever $\left(\geq 38.5{ }^{\circ} \mathrm{C}\right)$, laboratory values, chest $\mathrm{x}$-ray, and medication. To assess disease severity, the PSI [32] and the CURB-65 score [29] —validated risk assessment tools to categorize patients with CAP into different risk classes-were calculated upon ED admission. Confusion was also assessed as part of these scores. Discharge decision concerning patients enrolled in the study was left completely to the treating physicians without interference of the study team. For the present study, 285 patients with a final diagnosis of CAP and available serum blood specimens $[27,31]$ were included.

\section{Analysis of blood biomarker}

Within the initial trial, blood samples from each patient were collected and frozen upon ED admission for later 
measurement of different biomarkers. Cortisol, 11deoxycortisol, cortisone, and corticosterone levels were measured in admission serum blood samples of all 285 included patients with CAP.

After internal validation studies, we determined concentrations of selected hormones using a commercially available kit (MassChrom Steroids; Chromsystems, Munich, Germany). The analysis was performed using the UltiMate 3000 ultra-high-performance liquid chromatography (UHPLC) system (Thermo Fisher Scientific, San Jose, CA, USA) coupled to an AB Sciex 5500 quadrupole mass spectrometer (AB Sciex, Darmstadt, Germany). The Turbo V ion source (AB Sciex) was operated in positive electrospray ionization mode. The targeted screening method employed the multiple reaction monitoring mode of operation using two transitions for each analysis sample. Prior to injection into the UHPLC system, serum samples were subjected to a complex process of reversed phase 96-well solid-phase extraction, purification, and concentration as described in the MassChrom Steroids user's manual. Quantification of selected metabolites was achieved by reference to appropriate internal standards. Concentrations of all analyzed metabolites were reported in nanomoles per liter.

\section{Main outcome measurements}

The primary endpoint of this study was defined as 6-year all-cause mortality. As secondary endpoints, we reported mortality at days $30,60,90,180,240$, and 300 , as well as at 1 year, 2 years, and 3 years. Additional secondary endpoints were 30-day adverse outcome (including all-cause mortality and/or admission to the intensive care unit [ICU]) and disease severity defined by the PSI on admission. The decision for ICU admission was left to the treating physicians.

To validate outcomes, we performed blinded, structured telephone interviews at 30, 180, and 540 days after enrollment, as well as after a median of 6.1 years (IQR 5.6-6.5) [33, 34]. In cases where patients or their family members could not be contacted, the treating general practitioner was contacted.

\section{Statistical analyses}

Statistical analyses were conducted using STATA 12.1 software (StataCorp, College Station, TX, USA). $p$ Values $<0.05$ indicated statistical significance. Description of the study population was performed with descriptive statistics, including median with IQR to express continuous variables and frequency (percent) for categorical variables. The Wilcoxon rank-sum test was used for two-group comparison, whereas frequency comparison was done by chi-square test. All analyses were performed in the overall population.

Correlation analyses of glucocorticoid levels with inflammatory markers were calculated by Spearman's rank correlation. For multigroup comparisons, the Kruskal-Wallis test was performed. Univariate and multivariate Cox regression models were performed to investigate associations between glucocorticoid levels and all-cause mortality at different time points; these associations were reported as $\mathrm{HR}$ with 95\% CI. Before being entered into regression models, blood biomarker levels were log-transformed with a base of 10 due to a skewed distribution; after logarithmic transformation, the data approximated a normal distribution. Because of this transformation, HRs and ORs correspond to a tenfold increase in hormone levels. Kaplan-Meier curves were used to illustrate mortality based on glucocorticoid quartiles (highest versus lower three). To assess the association of glucocorticoid levels with other short-term adverse outcomes, we used univariate and multivariate logistic regression models, reported as OR with $95 \%$ CI. Significance levels were calculated by chi-square (Wald) test.

Models were adjusted for age, sex, and comorbidities (coronary heart disease, cerebrovascular insult, chronic kidney disease, neoplastic disease). The multivariate adjustment was predefined on the basis of factors known to be associated with mortality (age and comorbidities). We also included sex in the model because of expected differences in adrenal hormone levels based on gender. Initial randomization was not significantly associated with study endpoints and was thus not further considered for the statistical analysis.

\section{Results}

\section{Patient population}

Among a total of 285 patients with CAP, 135 (47.3\%) died during the 6-year follow-up period. The median age of the entire cohort was 71 years, and $60.4 \%$ of patients were male. There was a high burden of comorbidities, with $20.7 \%(n=59)$ of patients having underlying coronary heart disease, $15.4 \% \quad(n=44)$ having congestive heart failure, 23.5\% $(n=67)$ having chronic renal failure, 19.3\% $(n=55)$ having diabetes mellitus, and $13.3 \%(n=38)$ having neoplastic disease. We had blood levels available for cortisol in 227 patients (80\%), for 11-deoxycortisol in 249 patients (87\%), for cortisone in 283 patients (99\%), and for corticosterone in 285 patients (100\%). Additional baseline characteristics of the entire cohort, stratified by the primary endpoint as well as by 30-day adverse outcome, are shown in Table 1 and Additional file 1: Table S1, respectively.

Importantly, compared with the study population of the initial trial [26], including 925 patients with CAP with an all-cause mortality of $45 \%$ over a follow-up period of 6 years, the present analyzed cohort displayed a similar pattern of baseline characteristics [34]. Mortality in this cohort was $5.3 \%$ after 30 days and increased to $20.7 \%$ and $47.3 \%$ after 3 years and 6 years, respectively.

\section{Time-dependent association between glucocorticoid levels and adverse outcome}

Table 2 illustrates associations between admission serum glucocorticoid levels and all-cause mortality in a time- 
Table 1 Baseline characteristics overall and stratified by 6-year vital status in community-acquired pneumonia

\begin{tabular}{|c|c|c|c|c|}
\hline \multirow[b]{2}{*}{ Characteristics } & \multirow[b]{2}{*}{ Entire cohort $(n=285)$} & \multicolumn{3}{|l|}{ 6-Year vital status } \\
\hline & & Survivors $(n=150)$ & Nonsurvivors $(n=135)$ & $p$ Value \\
\hline \multicolumn{5}{|l|}{ Demographic characteristics } \\
\hline Age, years & $71[57-81]$ & $64[45-75]$ & 79 [70-84] & $<0.001$ \\
\hline Male sex & $172(60.4 \%)$ & $80(53.3 \%)$ & $92(68.1 \%)$ & 0.011 \\
\hline \multicolumn{5}{|l|}{ CAP characteristics } \\
\hline \multicolumn{5}{|l|}{ PSI class } \\
\hline । & $32(11.2 \%)$ & $30(20.0 \%)$ & $2(1.5 \%)$ & $<0.001$ \\
\hline$\|$ & $55(19.3 \%)$ & $44(29.3 \%)$ & $11(8.1 \%)$ & $<0.001$ \\
\hline III & $52(18.2 \%)$ & $29(19.3 \%)$ & $23(17.0 \%)$ & 0.62 \\
\hline IV & $104(36.5 \%)$ & $38(25.3 \%)$ & $66(48.9 \%)$ & $<0.001$ \\
\hline V & $42(14.7 \%)$ & $9(6.0 \%)$ & $33(24.4 \%)$ & $<0.001$ \\
\hline \multicolumn{5}{|l|}{ CURB-65 score } \\
\hline 0 & $63(22.1 \%)$ & $51(34.0 \%)$ & $12(8.9 \%)$ & $<0.001$ \\
\hline । & $67(23.5 \%)$ & $41(27.3 \%)$ & $26(19.3 \%)$ & 0.11 \\
\hline$\|$ & $82(28.8 \%)$ & $33(22.0 \%)$ & $49(36.3 \%)$ & 0.008 \\
\hline III & $57(20.0 \%)$ & $21(14.0 \%)$ & $36(26.7 \%)$ & 0.008 \\
\hline IVN & $16(5.6 \%)$ & $4(2.7 \%)$ & $12(8.9 \%)$ & 0.023 \\
\hline \multicolumn{5}{|l|}{ Comorbidities $^{a}$} \\
\hline Coronary heart disease & $59(20.7 \%)$ & $16(10.7 \%)$ & $43(31.9 \%)$ & $<0.001$ \\
\hline Congestive heart failure & $44(15.4 \%)$ & $7(4.7 \%)$ & $37(27.4 \%)$ & $<0.001$ \\
\hline Cerebrovascular insult & $28(9.8 \%)$ & $9(6.0 \%)$ & $19(14.1 \%)$ & 0.022 \\
\hline PAOD & $17(6.0 \%)$ & $7(4.7 \%)$ & $10(7.4 \%)$ & 0.33 \\
\hline Chronic renal failure & $67(23.5 \%)$ & $19(12.7 \%)$ & $48(35.6 \%)$ & $<0.001$ \\
\hline Diabetes mellitus & $55(19.3 \%)$ & $22(14.7 \%)$ & $33(24.4 \%)$ & 0.037 \\
\hline Neoplastic disease & $38(13.3 \%)$ & $12(8.0 \%)$ & $26(19.3 \%)$ & 0.005 \\
\hline \multicolumn{5}{|l|}{ Clinical history } \\
\hline Fever & 185 (65.1\%) & $113(75.3 \%)$ & $72(53.7 \%)$ & $<0.001$ \\
\hline Chills & 87 (34.0\%) & $58(42.0 \%)$ & $29(24.6 \%)$ & 0.003 \\
\hline Glucocorticoid pretreatment & $22(7.9 \%)$ & $5(3.4 \%)$ & $17(12.9 \%)$ & 0.003 \\
\hline \multicolumn{5}{|l|}{ Clinical findings } \\
\hline Confusion & $20(7.9 \%)$ & $4(2.9 \%)$ & $16(13.7 \%)$ & 0.002 \\
\hline Body temperature, ${ }^{\circ} \mathrm{C}$ & 38 [37.2-38.8] & $38.2[37.4-39]$ & $37.8[37-38.8]$ & 0.085 \\
\hline Breath rate, breaths/minute & 20 [16-25] & 20 [16-24] & 24 [18-28] & 0.002 \\
\hline Heart rate, beats/minute & 94 [82-105] & $92.5[83.5-108]$ & 95 [80-104] & 0.43 \\
\hline $\mathrm{SBP}, \mathrm{mmHg}$ & 130 [117-148] & 130 [120-148] & 130 [110-149] & 0.17 \\
\hline Arterial pH & $7.46[7.42-7.49]$ & $7.46[7.43-7.50]$ & $7.45[7.41-7.49]$ & 0.013 \\
\hline SIRS criteria & $188(66.0 \%)$ & $93(62.0 \%)$ & $95(70.4 \%)$ & 0.14 \\
\hline \multicolumn{5}{|l|}{ Outcome parameters } \\
\hline ICU admission & $21(7.4 \%)$ & $9(6.0 \%)$ & $12(8.9 \%)$ & 0.35 \\
\hline Mechanical ventilation & $7(2.5 \%)$ & $2(1.3 \%)$ & $5(3.7 \%)$ & 0.20 \\
\hline Septic shock & $6(2.1 \%)$ & $1(0.7 \%)$ & $5(3.7 \%)$ & 0.075 \\
\hline Length of stay, days & $8[5-12]$ & $7[4-10]$ & $9[6-13]$ & $<0.001$ \\
\hline \multicolumn{5}{|l|}{ Admission laboratory findings } \\
\hline CRP, mg/L & 132 [65-252] & $147[91-265]$ & 111 [55-249] & 0.037 \\
\hline
\end{tabular}


Table 1 Baseline characteristics overall and stratified by 6-year vital status in community-acquired pneumonia (Continued)

\begin{tabular}{lllll}
\hline $\mathrm{PCT}, \mu \mathrm{g} / \mathrm{L}$ & $0.48[0.16-3.20]$ & $0.64[0.16-3.72]$ & $0.43[0.16-1.97]$ & 0.37 \\
Cortisol, $\mathrm{nmol} / \mathrm{L}$ & $402[203.8-723.2]$ & $431[189.1-759.2]$ & $399[213.1-710.3]$ & 0.46 \\
11-Deoxycortisol, $\mathrm{nmol} / \mathrm{L}$ & $0.6[0.17-2.23]$ & $0.4[0.16-2.13]$ & $0.8[0.18-2.25]$ & 0.49 \\
Cortisone, nmol/L & $32.5[18.42-46.72]$ & $33.2[19.28-48.49]$ & $32.4[17.68-44.41]$ & 0.29 \\
Corticosterone, $\mathrm{nmol} / \mathrm{L}$ & $8.7[2.81-25.02]$ & $8.9[2.73-28.67]$ & $8.4[3.13-22.95]$ & 0.85
\end{tabular}

Abbreviations: CAP Community-acquired pneumonia, CRP C-reactive protein, CURB-65 Confusion of new onset, blood urea nitrogen $>7$ mmol/L, respiratory rate $\geq 30$ breaths per minute, systolic blood pressure $<90 \mathrm{mmHg}$ or diastolic blood pressure $\leq 60$, and age $\geq 65$ years, $P A O D$ Peripheral arterial occlusive disease, $P C T$ Procalcitonin, PSI Pneumonia severity index, SBP Systolic blood pressure, SIRS Systemic inflammatory response syndrome, ICU Intensive care unit Data are presented as median [IQR] or number (percent); $p<0.05$ is considered statistically significant. Bold values indicate statistical significance ${ }^{a}$ Comorbidities were identified on the basis of medical records or patient report

dependent manner. In the short term, all glucocorticoid hormones had HRs $>1$ and thus tended to be associated with higher mortality risk, but these associations changed over time. We found high initial cortisol and cortisone levels to be significantly associated with lower risk for 6-year mortality with HRs of 0.63 (95\% CI 0.41-0.99; $p=0.045$ ) and 0.60 (95\% CI $0.38-0.95 ; p=0.030$ ), respectively. For 3-year mortality, results were similar with HRs of 0.54 (95\% CI $0.32-0.90 ; p=0.018)$ and $0.56(95 \%$ CI $0.33-0.94 ; p=0.028)$ for cortisol and cortisone, respectively. For cortisol, these associations remained robust after adjustment for age, sex, and comorbidities. These results are also presented in Fig. 1, where a stepwise decrease in HRs with longer observation time for cortisol and cortisone, and to a lesser extent also for 11- deoxycortisol and corticosterone, can be observed. We further show Kaplan-Meier curves for 6-year survival stratified by glucocorticoid-level quartiles (Fig. 2). The highest quartile of admission cortisol and cortisone levels was favorably associated with long-term survival.

Also, high initial levels of cortisol were independently associated with higher risk for short-term adverse outcome (defined as risk for mortality and/or ICU admission) with an adjusted OR of 3.85 (95\% CI 1.10-13.49; $p=0.035$ ) (Table 3).

\section{Association between glucocorticoid levels and severity of CAP}

Multigroup comparison using the Kruskal-Wallis test showed a gradual increase in initial 11-deoxycortisol levels

Table 2 Association of admission glucocorticoid levels with short- and long-term all-cause mortality in community-acquired pneumonia

\begin{tabular}{|c|c|c|c|c|c|c|}
\hline \multirow[t]{3}{*}{ Entire cohort $(N=285)$} & \multicolumn{5}{|c|}{ All-cause mortality time point } & \multirow[b]{3}{*}{$p$ Value } \\
\hline & \multicolumn{2}{|l|}{30 days } & \multicolumn{2}{|l|}{3 years } & \multirow{2}{*}{$\begin{array}{l}6 \text { years } \\
\text { HR }(95 \% \mathrm{Cl})\end{array}$} & \\
\hline & $\mathrm{HR}(95 \% \mathrm{Cl})$ & $p$ Value & $\mathrm{HR}(95 \% \mathrm{Cl})$ & $p$ Value & & \\
\hline \multicolumn{7}{|l|}{ Cortisol } \\
\hline \multicolumn{7}{|l|}{ Cox regression analyses } \\
\hline Univariate model & 2.47 (95\% Cl 0.50-12.18) & 0.268 & $0.54(95 \%$ Cl 0.32-0.9) & 0.018 & $0.63(95 \%$ Cl 0.41-0.99) & 0.045 \\
\hline Multivariate model $\left.\right|^{a}$ & $1.93(95 \%$ Cl 0.51-9.02) & 0.405 & $0.53(95 \%$ Cl 0.32-0.89) & 0.017 & $0.57(95 \% \mathrm{Cl} 0.36-0.90)$ & 0.015 \\
\hline \multicolumn{7}{|l|}{ 11-Deoxycortisol } \\
\hline \multicolumn{7}{|l|}{ Cox regression analyses } \\
\hline Univariate model & 1.37 (95\% Cl 0.75-2.53) & 0.309 & 0.95 (95\% Cl 0.76-1.18) & 0.631 & $1.04(95 \%$ Cl 0.86-1.24) & 0.710 \\
\hline Multivariate model $^{a}$ & $1.26(95 \%$ Cl 0.65-2.44) & 0.486 & $0.84(95 \%$ Cl 0.67-1.05) & 0.126 & $0.91(95 \% \mathrm{Cl} 0.75-1.1)$ & 0.336 \\
\hline \multicolumn{7}{|l|}{ Cortisone } \\
\hline \multicolumn{7}{|l|}{ Cox regression analyses } \\
\hline Univariate model & 1.37 (95\% Cl 0.31-6.02) & 0.677 & $0.56(95 \%$ Cl 0.33-0.94) & 0.028 & $0.6(95 \%$ Cl 0.38-0.95) & 0.030 \\
\hline Multivariate model $^{a}$ & 2.55 (95\% Cl 0.38-17.30) & 0.337 & $0.71(95 \% \mathrm{Cl} 0.4-1.25)$ & 0.237 & $0.76(95 \% \mathrm{Cl} 0.46-1.24)$ & 0.272 \\
\hline \multicolumn{7}{|l|}{ Corticosterone } \\
\hline \multicolumn{7}{|l|}{ Cox regression analyses } \\
\hline Univariate model & $1.33(95 \%$ Cl 0.63-2.81) & 0.457 & 0.77 (95\% Cl 0.57-1.03) & 0.082 & $0.85(95 \%$ Cl 0.66-1.09) & 0.199 \\
\hline Multivariate model ${ }^{a}$ & $1.21(95 \%$ Cl $0.55-2.65)$ & 0.637 & $0.76(95 \%$ Cl 0.57-1.02) & 0.067 & $0.81(95 \% \mathrm{Cl} 0.63-1.03)$ & 0.084 \\
\hline
\end{tabular}

Data for univariate and multivariate Cox regression models are presented as $\mathrm{HR}(95 \% \mathrm{Cl}), p$ value; $p<0.05$ is considered statistically significant. Bold values indicate statistical significance. All hormone levels were log-transformed, and thus the HR corresponds to a tenfold increase in these levels

${ }^{a}$ Multivariate model is adjusted for age, sex, and comorbidities (coronary artery disease, cerebrovascular disease, chronic kidney disease, neoplastic disease) 


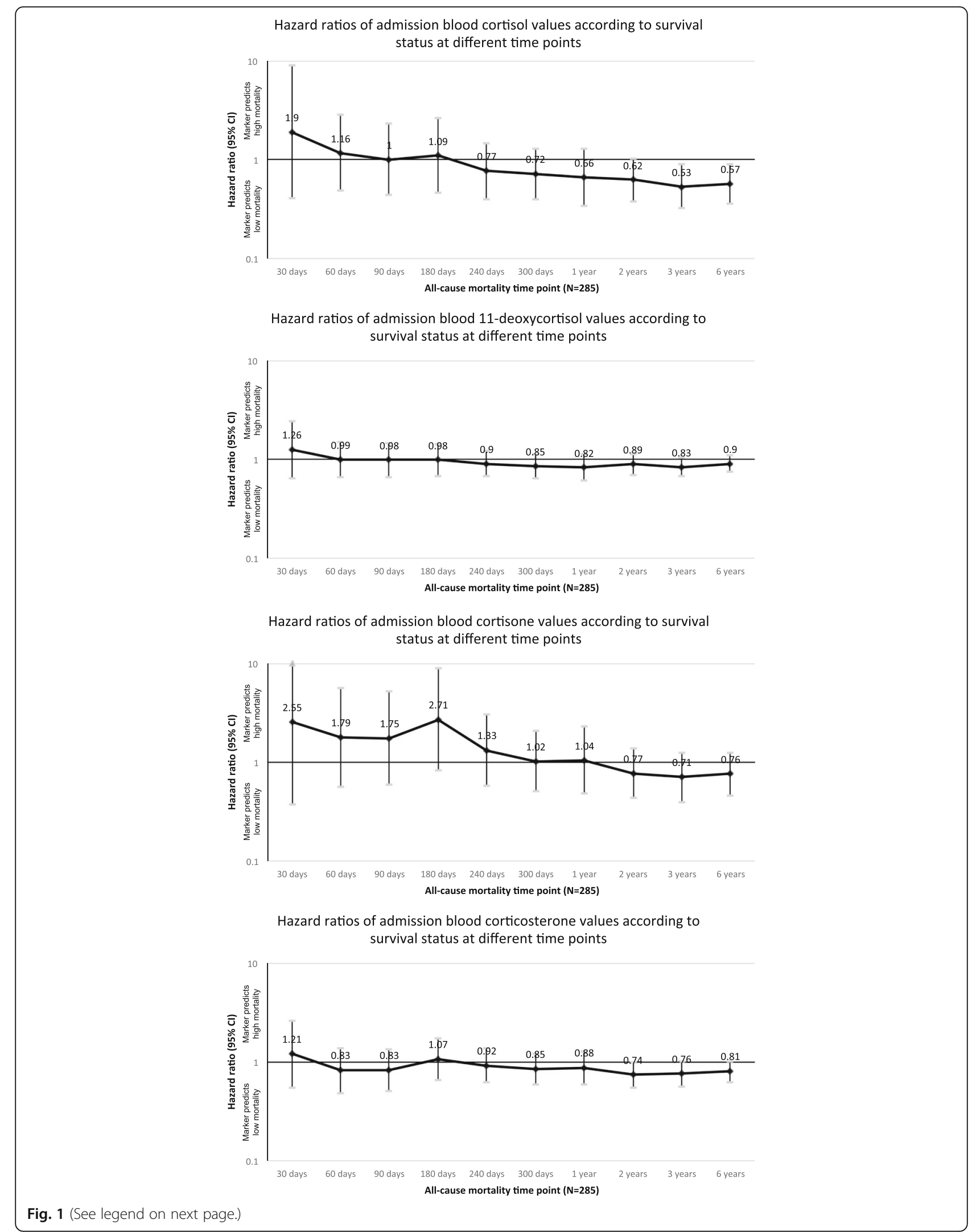


(See figure on previous page.)

Fig. 1 Association of admission glucocorticoid levels with all-cause mortality at different time points in CAP. Data for multivariate Cox regression models are presented as HR $(95 \% \mathrm{Cl})$. HRs $>1$ reflect a positive association between glucocorticoid levels and all-cause mortality. Multivariate model is adjusted for age, sex, and comorbidities (coronary artery disease, cerebrovascular disease, chronic kidney disease, neoplastic disease). CAP Community-acquired pneumonia

with increasing CAP severity as assessed by PSI classes $(p=0.001)$ (illustrated in Fig. 3). Contrarily, there was no similar increase in levels of cortisol, cortisone, and corticosterone with PSI. However, for cortisol, there was a trend of increasing hormone levels in more severely ill patients.

\section{Correlation between glucocorticoid levels and inflammatory markers}

As presented in Fig. 4, we found a correlation between admission cortisol levels and high peak CRP $(r=+0.22$; $p$ $=0.001)$ and initial PCT values $(r=+0.31 ; p<0.001)$. Cortisone levels were also positively correlated with CRP levels $(r=+0.14 ; p=0.023)$, whereas 11-deoxycortisol and corticosterone were significantly correlated with PCT levels $(r=+0.30$ and +0.20 , respectively; $p<0.001$ for both).

\section{Discussion}

The key findings of our present study of the prognostic value of different glucocorticoid hormones over a follow-up period of 6 years in patients with CAP are threefold. First, among different glucocorticoid hormones, cortisol showed the highest association with outcome in the short and long term. Second, the association of different glucocorticoid metabolites with adverse outcome inversely changed over time; whereas high levels of cortisol on initial hospital presentation were independently associated with short-term adverse outcome, a more pronounced initial glucocorticoid stress response was associated with favorable long-term outcome. Third, admission levels of 11-deoxycortisol showed the best correlation with disease severity.

The HPA stress axis plays an important role in patients with CAP, with cortisol being associated with short-term outcome [20-24] and corticosteroid treatment that might result in earlier time to recovery [35]. The release of cortisol in acute illness is essential for improvement in hemodynamic status, acute supply of energy, and protection against excessive inflammatory reaction $[5,6]$. Importantly, the acute stress response involves activation of the glucocorticoid pathway and the adrenergic system, resulting in tachycardia, increased myocardial oxygen consumption, and probably enhanced platelet aggregation, which might translate into acute myocardial infarction, thus potentially raising short-term mortality [18]. Owing to its positive correlation with the degree of illnessinduced stress [36], cortisol has been presumed to be a prognostic marker mirroring characteristics of acute critical disease and disease progression. Considering cortisol as the strongest inhibitor of inflammation, a higher level of proinflammatory cytokines in acute illness could lead to a more pronounced increase in cortisol [1-3, 37]. On the basis of several clinical observational studies [20-24],
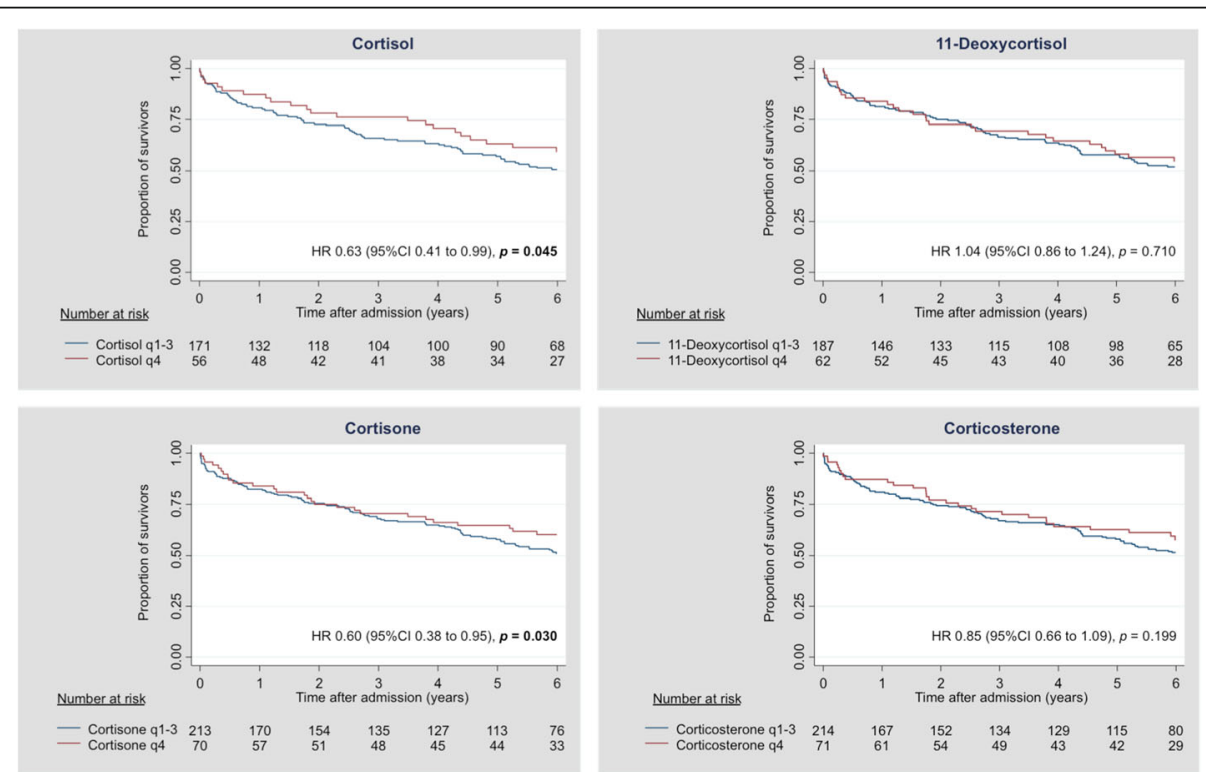

Fig. 2 Kaplan-Meier 6-year survival estimate according to admission glucocorticoid levels in CAP: fourth versus first-to-third quartiles. CAP Community-acquired pneumonia 
Table 3 Association of admission glucocorticoid levels with short-term adverse outcome in community-acquired pneumonia

\begin{tabular}{|c|c|c|}
\hline \multirow[t]{2}{*}{ Entire cohort $(N=285)$} & \multicolumn{2}{|c|}{ Adverse outcome at 30 days (death and/or ICU admission) } \\
\hline & OR $(95 \% \mathrm{Cl})$ & $p$ Value \\
\hline \multicolumn{3}{|l|}{ Cortisol } \\
\hline \multicolumn{3}{|c|}{ Logistic regression analyses } \\
\hline Univariate model & $4.16(95 \% \mathrm{Cl} 1.22-14.12)$ & 0.022 \\
\hline Multivariate model $^{\mathrm{a}}$ & $3.85(95 \%$ Cl 1.10-13.49) & 0.035 \\
\hline \multicolumn{3}{|l|}{ 11-Deoxycortisol } \\
\hline \multicolumn{3}{|c|}{ Logistic regression analyses } \\
\hline Univariate model & $1.33(95 \%$ Cl 0.84-2.11) & 0.216 \\
\hline Multivariate model $\left.\right|^{a}$ & $1.30(95 \%$ Cl $0.80-2.14)$ & 0.293 \\
\hline \multicolumn{3}{|l|}{ Cortisone } \\
\hline \multicolumn{3}{|c|}{ Logistic regression analyses } \\
\hline Univariate model & $1.89(95 \%$ Cl 0.59-1.03) & 0.280 \\
\hline Multivariate model $\left.\right|^{a}$ & $3.25(95 \%$ Cl 0.84-12.68) & 0.089 \\
\hline \multicolumn{3}{|l|}{ Corticosterone } \\
\hline \multicolumn{3}{|c|}{ Logistic regression analyses } \\
\hline Univariate model & $1.41(95 \% \mathrm{Cl} 0.81-2.47)$ & 0.225 \\
\hline Multivariate model $^{\mathrm{a}}$ & 1.44 (95\% Cl 0.79-2.62) & 0.234 \\
\hline
\end{tabular}

ICU, Intensive care unit

Data for univariate and multivariate logistic regression models are presented as $\mathrm{OR}(95 \% \mathrm{Cl}), p$ value; $p<0.05$ is considered statistically significant. Bold values indicate statistical significance. All hormone levels were log-transformed, and thus the OR corresponds to a tenfold increase in levels

${ }^{a}$ Multivariate model is adjusted for age, sex, and comorbidities (coronary artery disease, cerebrovascular disease, chronic kidney disease, neoplastic disease)

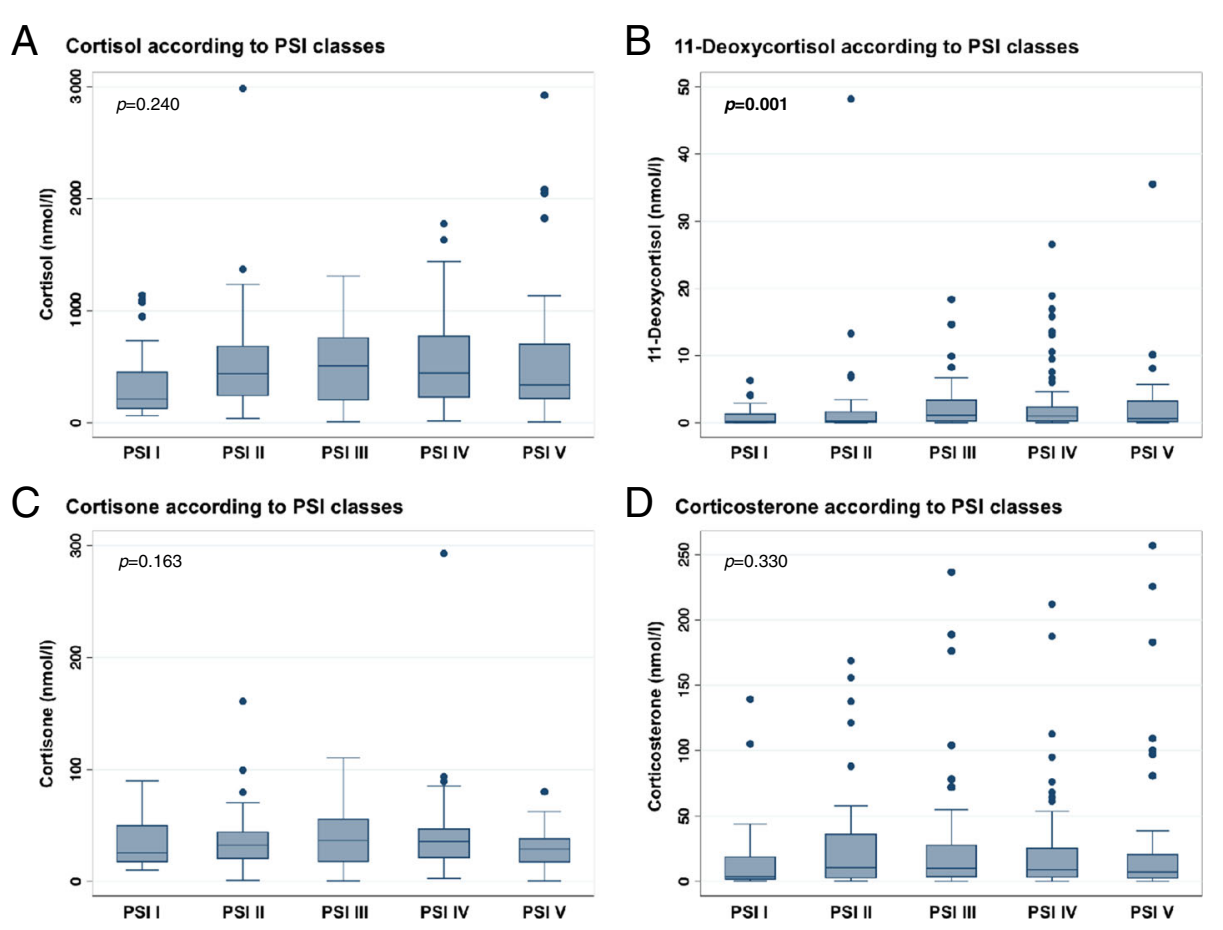

Fig. 3 Glucocorticoid levels in patients with various severity classes of CAP. Data represent median and IQR, with scatter plots presenting all values. $P$ values are determined by Kruskal-Wallis test and considered statistically significant at $p<0.05$. Bold values indicate statistical significance. CAP Community-acquired pneumonia, PSI Pneumonia severity index 

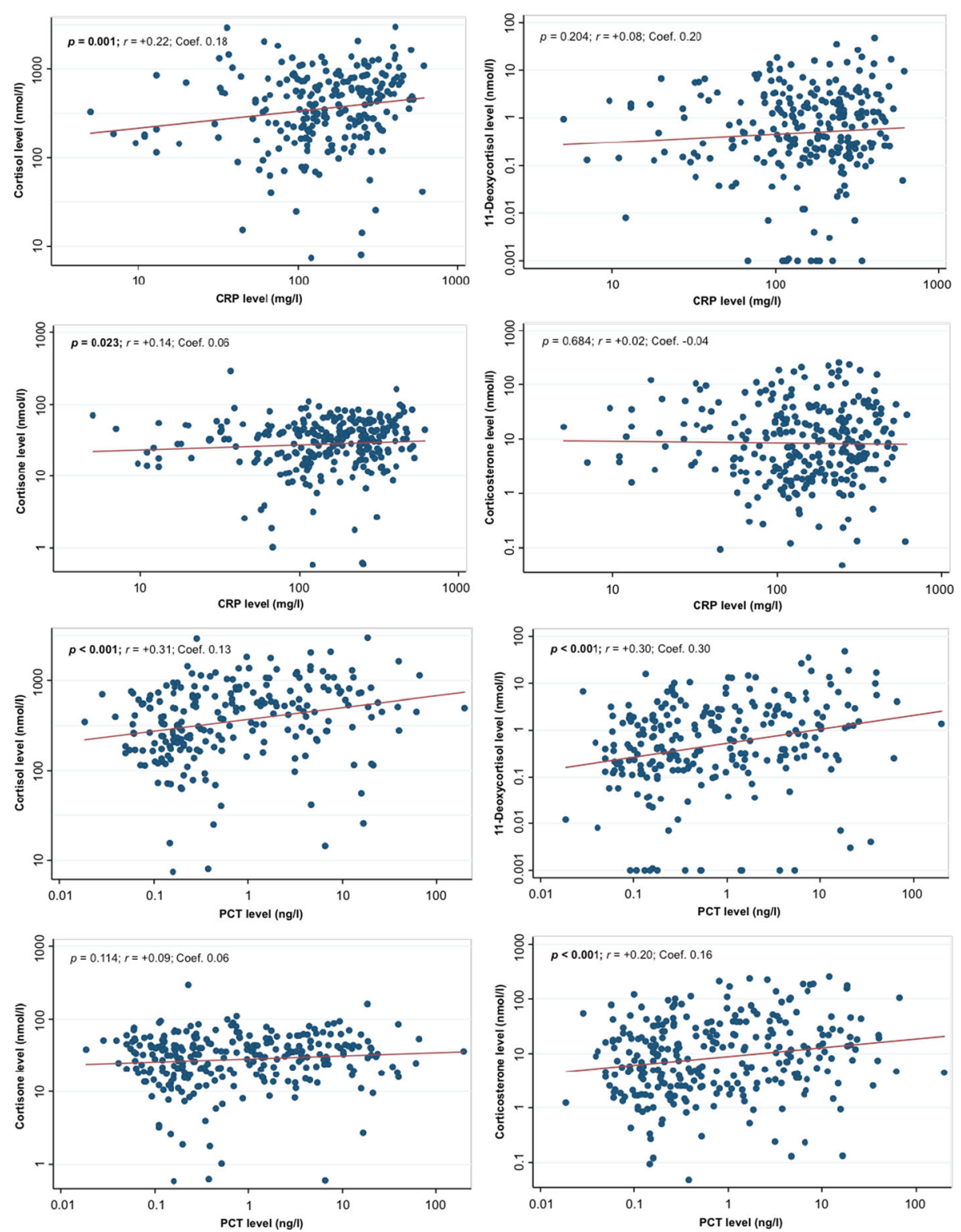

Fig. 4 Correlation of admission glucocorticoid levels with inflammatory markers in CAP. Data are presented with scatterplots showing all values (blue), overlaid by linear fit lines (red). Correlation analyses were performed by Spearman's rank correlation ( $r ; p$ value). We used multivariate linear regression models to calculate regression coefficients (Coef.). $p<0.05$ is considered statistically significant. Bold values indicate statistical significance. We used admission glucocorticoid and PCT levels and high peak CRP levels. CAP Community-acquired pneumonia, CRP C-reactive protein, PCT Procalcitonin. *Multivariate model is adjusted for age, sex, and comorbidities (coronary artery disease, cerebrovascular disease, chronic kidney disease, neoplastic disease)

cortisol levels have lately emerged as a useful prognostic tool regarding short-term mortality. The present analysis of a large, well-characterized cohort provides novel insights into the role of the glucocorticoid pathway in regard to adverse outcome in hospitalized patients with mild to severe CAP.

Regarding short-term outcome, our findings are in line with previous data derived from clinical observational studies suggesting that high levels of cortisol ae associated with mortality in CAP [20-24]. In our study, high admission cortisol levels were independently associated with short-term adverse outcome, including the combined endpoint mortality/ICU admission. Association of cortisol levels with 30-day all-cause mortality alone showed a similar trend; likely due to the small event number, the results did not reach statistical significance.

Interestingly, a more pronounced initial glucocorticoid host response was beneficial for long-term survival. For 
cortisol, this effect remained robust after adjustment for potential confounders. Because most previous studies have demonstrated a strong HPA stress axis response to be positively associated with adverse short-term outcomes [20-24], the present findings are somewhat counterintuitive. Hypothesizing that high levels of proinflammatory cytokines lead to an increase in cortisol $[1-3,37]$ to avoid excessive inflammation, a more pronounced initial glucocorticoid response might reflect not only the degree of stress but also the ability to generate an adequate immune response. Within this study, we demonstrated an independent correlation between admission cortisol levels and markers of inflammation (PCT and high peak CRP). From this point of view, our findings are in line with those of a previous study demonstrating a more pronounced inflammatory host response reflected by a history of chills, high body temperature, and high peak levels of CRP being associated with lower long-term mortality in patients surviving a CAP episode [38]. These results were similar to those of the Pneumonia Patient Outcomes Research Team cohort study, another large follow-up study of patients with CAP, in which researchers reported an association between the absence of fever and higher long-term mortality than among age-matched control subjects [39]. Contrarily, several studies have shown an association between worse short-term outcomes and strong inflammatory response in CAP [40-42]. On the basis of these data, it is tempting to hypothesize that survival of a clinically and biochemically pronounced CAP episode-reflected by high admission glucocorticoid levels-might mirror a more robust stress response, and, concordantly, also host defense, and thus a better general condition with lower mortality in the long term. However, a pronounced glucocorticoid response on admission might reflect, as a presumable consequence of inflammation, the ability to generate an appropriate immune response, thereby predisposing these "fitter" patients, with regard to the stress-response, to better long-term survival owing to decreased subsequent infections [43]. Further investigations are still warranted to validate these findings and determine underlying pathophysiology. Given the evidence of pronounced inflammation being associated with favorable long-term outcome in hospitalized patients with CAP, the rationale of steroid treatment in this population should be questioned.

The main strengths of this study include the wellcharacterized cohort of patients hospitalized for CAP, the long follow-up period of approximately 6 years, and the thorough and highly accurate biomarker measurement by liquid chromatography coupled with tandem mass spectrometry. This technique allows a proper separation and sensitive characterization of even small molecules [44]. Importantly, mass spectrometry is increasingly becoming accepted as a routine diagnostic instrument in clinical laboratories [45]. Furthermore, the large sample size and the high event number of the primary endpoint (47.3\%) lead to high statistical power, which allows detecting even small differences in glucocorticoid levels.

The following limitations require consideration. First, the present study is a secondary analysis and therefore not designed primarily to perform observational biomarker outcome studies. Importantly, there was no controlling for the time point of blood sampling, because initial blood samples were taken at the time of first patient contact in the ED. However, although cortisol exhibits diurnal concentration changes, during acute illness, the circadian pattern is usually lost $[5,46,47]$. Also, the long storage of blood may have affected blood hormone levels. In addition, there was no assessment of adrenal insufficiency based on the response to injection of synthetic adrenocorticotropin. Nevertheless, previous data demonstrated a very low rate of adrenal insufficiency in patients with CAP in the absence of septic shock [23]. However, deviation of the present results by different blood sampling time points or adrenal insufficiency cannot be definitively ruled out, although this lack of standardization reflects daily clinical practice. A more standardized hormone level determination at the same time of the day in all patients would most probably have demonstrated an even higher association between glucocorticoid levels and CAP outcomes. Moreover, serial hormone measurements over the course of the disease may add valuable information.

Second, the initial trial was conducted in different secondary and tertiary care hospitals in Switzerland, and thus results may not unconditionally be applied to other geographical or institutional settings. In addition, our analysis was focused specifically on patients with CAP; further studies are required to validate these findings for other medical and surgical patient populations. Finally, because this was an observational study, it is only hypothesis-generating, and correlation does not imply a causal relationship.

\section{Conclusions}

Cortisol showed the highest association with outcome in patients with mild to severe CAP. The association of the glucocorticoid levels with adverse outcome in patients with CAP changed over time; whereas high admission levels of cortisol were independently associated with short-term adverse outcome, a more pronounced initial glucocorticoid stress response was highly associated with favorable longterm outcome. Initial 11-deoxycortisol levels showed the highest correlation with disease severity. Underlying pathophysiological aspects are still poorly examined, and future studies are needed to better understand the importance of adrenal hormones in the resolution of patients with CAP. 


\section{Key messages}

- Among different glucocorticoid hormones, cortisol showed the highest association with mortality in patients with CAP.

- The association of glucocorticoid metabolites with outcome in CAP changed over time; whereas a more pronounced glucocorticoid stress response on initial hospital presentation was associated with higher short-term adverse outcome, increased admission hormone levels showed association with favorable long-term outcome.

\section{Additional file}

Additional file 1: Table S1. Baseline characteristics overall and stratified by 30-day adverse outcome, including the combined endpoint death/ICU admission in CAP. Data are presented as median [IQR] or number (percent); $p$ values are considered statistically significant at $p<0.05$. Bold values indicate statistical significance. CAP, Community-acquired pneumonia; CRP, C-reactive protein; CURB65, Confusion of new onset, blood urea nitrogen $>7 \mathrm{mmol} / \mathrm{L}$, respiratory rate $\geq 30$ breaths per minute, systolic blood pressure $<90 \mathrm{mmHg}$ or diastolic blood pressure $\leq 60$, and age $\geq 65$ years; ICU, Intensive care unit; PAOD, Peripheral arterial occlusive disease; PCT, Procalcitonin; PSI, Pneumonia severity index; SBP, Systolic blood pressure; SIRS, Systemic inflammatory response syndrome. ${ }^{*}$ Comorbidities were identified on the basis of medical records or patient report. (DOCX $21 \mathrm{~kb}$ )

\section{Abbreviations}

CAP: Community-acquired pneumonia; CRP: C-reactive protein; CURB65: Confusion of new onset, blood urea nitrogen $>7 \mathrm{mmol} / \mathrm{L}$, respiratory rate $\geq 30$ breaths per minute, systolic blood pressure $<90 \mathrm{mmHg}$ or diastolic blood pressure $\leq 60$, and age $\geq 65$ years; ED: Emergency department;

HPA: Hypothalamic-pituitary-adrenal; ICU: Intensive care unit; PAOD: Peripheral arterial occlusive disease; PCT: Procalcitonin; PSI: Pneumonia severity index; SBP: Systolic blood pressure; SIRS: Systemic inflammatory response syndrome; UHPLC: Ultra-high-performance liquid chromatography

\section{Acknowledgements}

We are grateful to the emergency department, medical clinic, and central laboratory staff of the University Hospital Basel and the cantonal hospitals of Aarau, Liestal, Lucerne, Muensterlingen, and the 'Buergerspital' Solothurn for their assistance and technical support. In particular, we thank all patients, their relatives, and all local general practitioners who participated in this study. Finally, we acknowledge the ProHOSP Study Group for their important support. The ProHOSP Study Group includes the following persons: Ursula Schild, RN; Katharina Regez, RN; Rita Bossart, RN; Robert Thomann, MD; Claudine Falconnier, MD; Marcel Wolbers, PhD; Stefanie Neidert, MD; Thomas Fricker, MD; Claudine Blum, MD; Thomas Bregenzer, MD; Claus Hoess, MD; Heiner C. Bucher, MD; Fabian Mueller; Jeannine Haeuptle; Roya Zarbosky; Rico Fiumefreddo, MD; Melanie Wieland, RN; Charly Nusbaumer, MD; Andres Christ, MD; Roland Bingisser, MD; Kristian Schneider, RN; Brigitte Walz, PhD; Verena Briner, MD; Dieter Conen, MD; Andreas Huber, MD; Jody Staehelin, MD; Chantal Bruehlhardt, RN; Ruth Luginbuehl, RN; Agnes Muehlemann, PhD; Ineke Lambinon; Werner Zimmerli, MD; and Max Zueger, MD.

\section{Funding}

This study was supported in part by the Swiss National Science Foundation (SNSF Professorship PP00P3_150531/1) and the Research Council of the Kantonsspital Aarau (1410.000.044). The initial trial was funded by the Swiss National Science Foundation (grant SNF 3200BO-116177/1), Santé Suisse, and the Gottfried and Julia Bangerter-Rhyner Foundation. The funding organization(s) played no role in the design of the study; in the collection, analysis, and interpretation of data; or in the writing of the manuscript.

\section{Availability of data and materials}

The datasets used and analyzed during the present study are available from the corresponding author on reasonable request.

\section{Authors' contributions \\ MCC, BM, and PS created the study concept and design, wrote the protocol, and initiated the initial ProHOSP study. MN and PS drafted the manuscript and performed statistical analyses. CS and AH performed laboratory measurements of glucocorticoids. All authors contributed to the data acquisition, interpretation and drafting of the analyses, as well as to critical review for important intellectual content, and final approval of the manuscript. PS had full access to all data in the present study and takes responsibility for the integrity of the work and the accuracy of the data analyses. $\mathrm{MO}, \mathrm{AK}, \mathrm{WZ}, \mathrm{CH}, \mathrm{CH}$, and $\mathrm{LB}$ contributed to the data acquisition, interpretation and drafting of the analyses, as well as to critical review for important intellectual content, and final approval of the manuscript.}

\section{Competing interests}

The authors declare that they have no competing interests.

\section{Consent for publication}

Not applicable.

\section{Ethics approval and consent to participate}

The study protocol was approved by the ethics committee of the University of Basel as well as by the local ethics committees of Aarau, Muensterlingen, Solothurn, and Luzern, all with the same ethical study number 87/06. Written informed consent was provided by all participants for the initial trial, including agreement to use their data anonymized in secondary analyses.

\section{Author details}

${ }^{1}$ Medical University Department, Division of General Internal and Emergency Medicine, Kantonsspital Aarau, Aarau, Switzerland. Department of Laboratory Medicine, Kantonsspital Aarau, Aarau, Switzerland. ${ }^{3}$ Division of Endocrinology, Diabetes and Clinical Nutrition, Department of Internal Medicine, University Hospital Basel, Basel, Switzerland. ${ }^{4}$ Medical Faculty, University of Basel, Basel, Switzerland. ${ }^{5}$ Basel University Medical Clinic Liestal, Liestal, Switzerland.

${ }^{6}$ Department of Internal Medicine, Bürgerspital Solothurn, Solothurn, Switzerland. ${ }^{7}$ Department of Internal Medicine, Kantonsspital Münsterlingen, Münsterlingen, Switzerland. ${ }^{8}$ Department of Internal Medicine, Kantonsspital Lucerne, Lucerne, Switzerland.

Received: 16 November 2016 Accepted: 28 February 2017

Published online: 24 March 2017

\section{References}

1. Chrousos GP. The hypothalamic-pituitary-adrenal axis and immunemediated inflammation. N Engl J Med. 1995;332(20):1351-62.

2. Nylen ES, Müller B. Endocrine changes in critical illness. J Intensive Care Med. 2004;19(2):67-82.

3. Müller B. Endocrine aspects of critical illness. Ann Endocrinol (Paris). 2007; 68(4):290-8.

4. Schuetz $P$, Müller B. The hypothalamic-pituitary-adrenal axis in critical illness. Endocrinol Metab Clin North Am. 2006:35(4):823-38.

5. Van den Berghe G, de Zegher F, Bouillon R. Clinical review 95: acute and prolonged critical illness as different neuroendocrine paradigms. J Clin Endocrinol Metab. 1998;83(6):1827-34.

6. Marik PE, Kiminyo K, Zaloga GP. Adrenal insufficiency in critically ill patients with human immunodeficiency virus. Crit Care Med. 2002;30(6):1267-73.

7. Jurney TH, Cockrell Jr JL, Lindberg JS, Lamiell JM, Wade CE. Spectrum of serum cortisol response to ACTH in ICU patients: correlation with degree of illness and mortality. Chest. 1987;92(2):292-5.

8. Span LF, Hermus AR, Bartelink AK, Hoitsma AJ, Gimbrere JS, Smals AG, et al. Adrenocortical function: an indicator of severity of disease and survival in chronic critically ill patients. Intensive Care Med. 1992;18(2):93-6.

9. Annane D, Sebille V, Troche G, Raphael JC, Gajdos P, Bellissant E. A 3-level prognostic classification in septic shock based on cortisol levels and cortisol response to corticotropin. JAMA. 2000;283(8):1038-45.

10. Sam S, Corbridge TC, Mokhlesi B, Comellas AP, Molitch ME. Cortisol levels and mortality in severe sepsis. Clin Endocrinol (Oxf). 2004;60(1):29-35. 
11. Burchard K. A review of the adrenal cortex and severe inflammation: quest of the "eucorticoid" state. J Trauma. 2001;51(4):800-14.

12. Schein RM, Sprung CL, Marcial E, Napolitano L, Chernow B. Plasma cortisol levels in patients with septic shock. Crit Care Med. 1990;18(3):259-63.

13. Arlt W, Hammer F, Sanning P, Butcher SK, Lord JM, Allolio B, et al. Dissociation of serum dehydroepiandrosterone and dehydroepiandrosterone sulfate in septic shock. J Clin Endocrinol Metab. 2006;91(7):2548-54.

14. Feng JY, Liu KT, Abraham E, Chen CY, Tsai PY, Chen YC, et al. Serum estradiol levels predict survival and acute kidney injury in patients with septic shock - a prospective study. PLoS One. 2014;9(6):e97967.

15. Kung HC, Hoyert DL, XU J, Murphy SL. Deaths: final data for 2005. Natl Vital Stat Rep. 2008;56(10):1-120.

16. Brown PD, Lerner SA. Community-acquired pneumonia. Lancet. 1998; 352(9136):1295-302.

17. File TM. Community-acquired pneumonia. Lancet. 2003;362(9400):1991-2001.

18. Santos-Gallego CG, Badimon JJ. The sum of two evils: pneumonia and myocardial infarction: is platelet activation the missing link? J Am Coll Cardiol. 2014;64(18):1926-8

19. Santos-Gallego CG, Badimon JJ. Cardiac complications after communityacquired pneumonia. Am J Cardiol. 2016;117(2):310.

20. Christ-Crain M, Stolz D, Jutla S, Couppis O, Müller C, Bingisser R, et al. Free and total cortisol levels as predictors of severity and outcome in communityacquired pneumonia. Am J Respir Crit Care Med. 2007;176(9):913-20.

21. Kolditz M, Hoffken G, Martus P, Rohde G, Schutte H, Bals R, et al. Serum cortisol predicts death and critical disease independently of CRB-65 score in community-acquired pneumonia: a prospective observational cohort study. BMC Infect Dis. 2012;12:90

22. Mueller C, Blum CA, Trummler M, Stolz D, Bingisser R, Mueller C, et al. Association of adrenal function and disease severity in community-acquired pneumonia. PLoS One. 2014;9(6):e99518.

23. Kolditz M, Halank M, Schulte-Hubbert B, Hoffken G. Adrenal function is related to prognosis in moderate community-acquired pneumonia. Eur Respir J. 2010;36(3):615-21.

24. Salluh Jl, Bozza FA, Soares M, Verdeal JC, Castro-Faria-Neto HC, Lapa e Silva JR, Bozza PT. Adrenal response in severe community-acquired pneumonia: impact on outcomes and disease severity. Chest. 2008;134(5):947-54.

25. Nickler M, Ottiger M, Steuer C, Huber A, Anderson JB, Müller B, et al. Systematic review regarding metabolic profiling for improved pathophysiological understanding of disease and outcome prediction in respiratory infections. Respir Res. 2015;16:125.

26. Schuetz P, Christ-Crain M, Thomann R, Falconnier C, Wolbers M, Widmer I, et al. Effect of procalcitonin-based guidelines vs standard guidelines on antibiotic use in lower respiratory tract infections: the ProHOSP randomized controlled trial. JAMA. 2009;302(10):1059-66.

27. Schuetz P, Christ-Crain M, Wolbers M, Schild U, Thomann R, Falconnier C, et al. Procalcitonin guided antibiotic therapy and hospitalization in patients with lower respiratory tract infections: a prospective, multicenter, randomized controlled trial. BMC Health Serv Res. 2007;7:102.

28. Gonzales R, Sande MA. Uncomplicated acute bronchitis. Ann Intern Med. 2000;133(12):981-91

29. Niederman MS, Mandell LA, Anzueto A, Bass JB, Broughton WA, Campbell $\mathrm{GD}$, et al. Guidelines for the management of adults with communityacquired pneumonia: diagnosis, assessment of severity, antimicrobial therapy, and prevention. Am J Respir Crit Care Med. 2001;163(7):1730-54.

30. Woodhead M, Blasi F, Ewig S, Huchon G, leven M, Ortqvist A, et al. Guidelines for the management of adult lower respiratory tract infections. Eur Respir J. 2005;26(6):1138-80.

31. Schuetz P, Christ-Crain M, Albrich W, Zimmerli W, Mueller B, ProHOSP Study Group. Guidance of antibiotic therapy with procalcitonin in lower respiratory tract infections: insights into the ProHOSP study. Virulence. 2010; 1(2):88-92. doi:10.4161/viru.1.2.10488.

32. Fine MJ, Auble TE, Yealy DM, Hanusa BH, Weissfeld LA, Singer DE, et al. A prediction rule to identify low-risk patients with community-acquired pneumonia. N Engl J Med. 1997;336(4):243-50.

33. Grolimund E, Kutz A, Marlowe RJ, Vogeli A, Alan M, Christ-Crain M, et al. Long-term prognosis in COPD exacerbation: role of biomarkers, clinical variables and exacerbation type. COPD. 2015;12(3):295-305.

34. Alan M, Grolimund E, Kutz A, Christ-Crain M, Thomann R, Falconnier C, et al. Clinical risk scores and blood biomarkers as predictors of long-term outcome in patients with community-acquired pneumonia: a 6-year prospective follow-up study. J Intern Med. 2015;278(2):174-84.
35. Blum CA, Nigro N, Briel M, Schuetz P, Ullmer E, Suter-Widmer I, et al. Adjunct prednisone therapy for patients with community-acquired pneumonia: a multicentre, double-blind, randomised, placebo-controlled trial. Lancet. 2015;385(9977):1511-8.

36. Arafah BM. Hypothalamic pituitary adrenal function during critical illness: limitations of current assessment methods. J Clin Endocrinol Metab. 2006; 91(10):3725-45.

37. Beishuizen A, Thijs LG. Endotoxin and the hypothalamo-pituitary-adrenal (HPA) axis. J Endotoxin Res. 2003;9(1):3-24.

38. Guertler C, Wirz B, Christ-Crain M, Zimmerli W, Mueller B, Schuetz P. Inflammatory responses predict long-term mortality risk in communityacquired pneumonia. Eur Respir J. 2011;37(6):1439-46.

39. Mortensen EM, Kapoor WN, Chang CC, Fine MJ. Assessment of mortality after long-term follow-up of patients with community-acquired pneumonia. Clin Infect Dis. 2003;37(12):1617-24

40. Almirall J, Bolibar I, Toran P, Pera G, Boquet X, Balanzo X, et al. Contribution of $C$-reactive protein to the diagnosis and assessment of severity of community-acquired pneumonia. Chest. 2004;125(4):1335-42.

41. Menendez R, Martinez R, Reyes S, Mensa J, Polverino E, Filella X, et al. Stability in community-acquired pneumonia: one step forward with markers? Thorax. 2009;64(11):987-92.

42. Seligman R, Meisner M, Lisboa TC, Hertz FT, Filippin TB, Fachel JM, et al. Decreases in procalcitonin and $\mathrm{C}$-reactive protein are strong predictors of survival in ventilator-associated pneumonia. Crit Care. 2006;10(5):R125.

43. Mortensen EM. Potential causes of increased long-term mortality after pneumonia. Eur Respir J. 2011:37(6):1306-7.

44. Steuer C, Schütz P, Bernasconi L, Huber AR. Simultaneous determination of phosphatidylcholine-derived quaternary ammonium compounds by a LCMS/MS method in human blood plasma, serum and urine samples. J Chromatogr B Analyt Technol Biomed Life Sci. 2016;1008:206-11.

45. Tenori L, Oakman C, Claudino WM, Bernini P, Cappadona S, Nepi S, et al. Exploration of serum metabolomic profiles and outcomes in women with metastatic breast cancer: a pilot study. Mol Oncol. 2012;6(4):437-44.

46. Van den Berghe $\mathrm{GH}$. Acute and prolonged critical illness are two distinct neuroendocrine paradigms. Verh K Acad Geneeskd Belg. 1998;60(6):487-520

47. Bartanusz V, Corneille MG, Sordo S, Gildea M, Michalek JE, Nair PV, et al. Diurnal salivary cortisol measurement in the neurosurgical-surgical intensive care unit in critically ill acute trauma patients. J Clin Neurosci. 2014;21(12):2150-4.

\section{Submit your next manuscript to BioMed Central and we will help you at every step:}

- We accept pre-submission inquiries

- Our selector tool helps you to find the most relevant journal

- We provide round the clock customer support

- Convenient online submission

- Thorough peer review

- Inclusion in PubMed and all major indexing services

- Maximum visibility for your research

Submit your manuscript at www.biomedcentral.com/submit
) Biomed Central 